\title{
PUBLICACIONES SOBRE LITERATURA FRANCESA EN ESPAÑA 2001
}

Alicla Yllera

UNED. Madrid

\section{Obras colectivas. Revistas}

Aliaga, Juan Vicente. Haderbache, Ahmed, Monleón. Ana, Pujante, Domingo (eds.), Miradas sobre la sexualidad en el arte y la literatura del siglo XX en Francia y España. Valencia: Servei de Publicacions de la Universitat de València, 2001, 650 pp.

BoixAREU, Mercedes, DESNÉ. Roland (eds.). Recepción de autores franceses de la época clásica en los siglos XVIII y XIX en España y en el extranjero. Madrid: UNED, 2001, 380 pp.

De Diego, Rosa, Vázquez, Lydia (eds.), La máquina escénica: drama, espacio, tecnología. Servicio de Publicaciones de la U.P.V./ E.H.U., 2001.

FERNÁNDEZ SÁNCHEZ. Carmen (ed.). Literatura francocanadiense: la literatura quehequesa. Oviedo: Universidad de Oviedo, Servicio de Publicaciones, 2001, 340 pp. González Salvador, Ana (ed.), Cuadernos de Filología Francesa. Balzac (Universidad de Extremadura) 13 (2001).

Gregori, Carmen, Jiménez, Dolores, Martínez Luciano, Juan Vicente (eds.), $\mathbf{H u}$ mor y Literatura. Quaderns de Filologia, Estudios Literaris, VI. Valencia: Facultat de Filologia, Universitat de València, 2001, $248 \mathrm{pp}$.

Lafarga, Francisco, Domínguez, Antonio (eds.). Los clásicos franceses en la España del siglo XX. Estudios de traducción y recepción. Barcelona: PPU, 2001, 277 pp. 
Real, Elena, Jiménez, Dolores, Pujante, Domingo, Cortuo, Adela (eds.), Écrire, traduire et représenter la fête. VIII Coloquio de la Asociación de Profesores de Filología Francesa de la Universidad Española. Valencia: Universitat de València, Facultat de Filologia/ A.P.F.F.U.E., 2001, 833 pp.

SANTA, Àngels, GinÉ, Marta (eds.), Surrealimo y literatura en Europa. Lleida: Edicions de la Universitat de Lleida, 2001, 202 pp.

TORRES MONREAL, Francisco (ed.), El teatro y lo sagrado: de $M$. de Ghelderode a $F$. Arrabal. Edición, Introducción, Coordinación y Epílogo. Murcia: Universidad de Murcia, Servicio de publicaciones, 2001, 689 pp.

\section{EDAD MEDIA}

ARAmburu Riera, Francisca, "Le nu et le vêtu d'une princesse sarrasine dans Fierabras», Senefiance (Université de Provence) 47 (2001): 7-16.

BENITO DE LA FUENTE, Javier, «Le nu et le vêtu dans les romans arthuriens du XIII siècle». Senefiance (Université de Provence) 47 (2001): 127-138. [Lancelot en prose].

Carmona Fernández, Fernando, La mentalidad literaria medieval. Murcia: Servicio de Publicaciones, 2001.

Desprès CAUbrière, Catherine, «Le nu sous le vêtu dans quelques extraits du Roman Antique», Senefiance (Université de Provence) 47 (2001): 105-114.

García PRADas, Ramón, «La fiesta de los locos. Un origen folklórico para el teatro del medievo francés», Écrire, traduire et représenter la fête. VIII Coloquio de la Asociación de Profesores de Filología Francesa de la Universidad Española, Real, Elena, Jiménez, Dolores, Pujante, Domingo, Cortijo, Adela (eds.). Valencia: Universitat de València, Facultat de Filologia/ A.P.F.F.U.E., 2001, pp. 33-41.

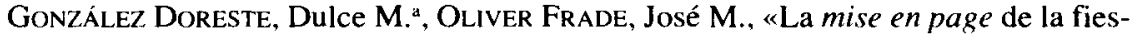
ta en dos manuscritos del Roman de la Rose», Écrire, traduire et représenter la fête. VIII Coloquio de la Asociación de Profesores de Filología Francesa de la Universidad Española, Real, Elena, Jiménez, Dolores, Pujante, Domingo, Cortijo, Adela (eds.). Valencia: Universitat de València, Facultat de Filologia/ A.P.F.F.U.E., 2001, pp. 57-70.

IÑARREA LAS HERAS, Ignacio, «Descripción de los itinerarios incluidos en los cantosguía franceses de la ruta jacobea», Estudios de Filología Moderna 2 (2001): 7-33.

INAARREA LAS HERAS, Ignacio, «El tema de la tempestad en las canciones de peregrinos franceses de la ruta jacobea", Écrire, traduire et représenter la fête. VIII Coloquio de la Asociación de Profesores de Filología Francesa de la Universidad Española. Real, Elena, Jiménez, Dolores, Pujante, Domingo, Cortijo, Adela (eds.). Valencia: Universitat de València, Facultat de Filologia/ A.P.F.F.U.E., 2001, pp. 89-102. 
LÓPEZ AlCARAZ, Josefa, «Excursus morales en los fabliaux: la expresión de la religiosidad medieval», Literatura y cristiandad, Homenaje a Jesús Montoya, Granada, 2001, pp. 63-76.

Martínez PÉrez, Antonia, «Adam de la Halle y Rutebeuf: sus lamentaciones personales», Estudios Románicos (Universidad de Murcia) 12 (2001): 209-224.

Morales PeCo, Monserrat, «Representación de la antigüedad clásica en el Roman de Thèbes: las celebraciones de la estirpe de Edipo», Écrire, traduire et représenter la fête. VIII Coloquio de la Asociación de Profesores de Filología Francesa de la Universidad Española. Real, Elena, Jiménez, Dolores, Pujante, Domingo, Cortijo, Adela (eds.). Valencia: Universitat de València, Facultat de Filologia/ A.P.F.F.U.E., 2001, pp. 43-56.

RiQuER, Isabel de, «Introducción, traducción y notas» a Tomás de Inglaterra, Berol, María de Francia y otros, Tristán e Iseo. Madrid: Ediciones Siruela, 2001.

Travieso Ganaza, Mercedes, "La fiesta del loco en el Jeu de la Feuillée», Écrire. traduire et représenter la fête. VIII Coloquio de la Asociación de Profesores de Filologia Francesa de la Universidad Española, Real, Elena, Jiménez, Dolores, Pujante, Domingo, Cortijo, Adela (eds.). Valencia: Universitat de València, Facultat de Filologia/ A.P.F.F.U.E., 2001, pp. 19-32.

\section{Siglo XVI}

BENOIT MORINIÈRE, Claude, «Du sourire ironique au rire farcesque. Les premiers pas de la nouvelle comédie: l'Eugène d'Étienne Jodelle», Humor y Literatura. Quaderns de Filologia, Estudios Literaris, VI, Gregori, Carmen, Jiménez, Dolores, Martínez Luciano, Juan Vicente (eds.). Valencia: Facultat de Filologia, Universitat de València, 2001, pp. 29-42

GonzÁlez AlCARAZ, José Antonio, «Étienne Jodelle y la tragedia renacentista», Estudios Románicos (Universidad de Murcia) 12 (2001): 91-100.

\section{Siglo XVII}

Benort Morinière, Claude, «Pour le plaisir des yeux: Les Amours de Psyché et de Cupidon de J. de La Fontaine», Queste (Le Regard) 9, Sampy, Joëlle (ed.) (2001): 35-45.

Pérez Pérez. Concepción, «Alrededor de una habitación. Del Viaje a la Expedición nocturna de Xavier de Maistre», Creación espacial y narración literaria, Pérez, Concepción, Caballos, M. ${ }^{a}$ de Gracia, Raventós, Anna (eds.). Sevilla: Universidad de Sevilla, Grupo de Investigación Temático Estructural, 2001, pp. 338-344. 
5. SIGLO XVIII

PUjANTE GonZÁleZ, Domingo, "Vénus et Thanatos: une vision masculine de l'amour au XVIII ${ }^{e}$ siècle», Estudios de Lengua y Literatura francesas (Universidad de Cádiz) 13 (2001): 151-180.

6. SIGLO XIX

Álvarez ORdóñez, Gemma, «Chateaubriand et les salons», Écrire, traduire et représenter la fête. VIII Coloquio de la Asociación de Profesores de Filología Francesa de la Universidad Española, Real, Elena, Jiménez, Dolores, Pujante, Domingo, Cortijo, Adela (eds.). Valencia: Universitat de València, Facultat de Filologia/ A.P.F.F.U.E., 2001, pp. 175-185.

BERMÚDEZ MEDINA, Dolores, «Une nature lointainement étrangère: l'androgyne dans Double de Francis Poictevin», Mythes de la décadence, Montandon, A. (ed.). Clermont-Ferrand: Presses Universitaires Blaise Pascal, 2001, pp. 219-228.

Caballos Bejano, M. ${ }^{a}$ de Gracia, «El dormitorio como espacio de ensoñación femenina en Nana», Creación espacial y narración literaria, Pérez, Concepción, Caballos, M. ${ }^{a}$ de Gracia, Raventós, Anna (eds.). Sevilla: Universidad de Sevilla, Grupo de Investigación Temático Estructural, 2001, pp. 141-147.

Campos Plaza, Nicolás, Campos Martín, Natalia, «El universo lúdico de Théophile Gautier en Voyage en Espagne. La Mancha», Écrire, traduire et représenter la fête. VIII Coloquio de la Asociación de Profesores de Filología Francesa de la Universidad Española, Real, Elena, Jiménez, Dolores, Pujante, Domingo, Cortijo, Adela (eds.). Valencia: Universitat de València, Facultat de Filologia/ A.P.F.F.U.E., 2001, pp. 197-207.

Delgado Cabrera, Arturo, Menéndez Ayuso, Emilio, «Saynète, opérette, fête: en torno a Mérimée y Offenbach», Écrire, traduire et représenter la fête. VIII Coloquio de la Asociación de Profesores de Filología Francesa de la Universidad Española, Real, Elena, Jiménez, Dolores, Pujante, Domingo, Cortijo, Adela (eds.). Valencia: Universitat de València, Facultat de Filologia/ A.P.F.F.U.E., 2001, pp. 145-155.

Gutiérrez Viñayo, Félix César, «Balzac, un toit pour l'éternité. De la Cabane de Passy à la Cathédrale littéraire, la fête continue», Écrire, traduire et représenter la fête. VIII Coloquio de la Asociación de Profesores de Filología Francesa de la Universidad Española, Real, Elena, Jiménez, Dolores, Pujante, Domingo, Cortijo, Adela (eds.). Valencia: Universitat de València, Facultat de Filologia/ A.P.F.F.U.E., 2001, pp. 209-220.

LEAL, Juli, «On nous a dit: soyez gais! o, en otras palabras, de la fête à la défaite», Écrire, traduire et représenter la fête. VIII Coloquio de la Asociación de Profe- 
sores de Filología Francesa de la Universidad Española, Real, Elena, Jiménez, Dolores, Pujante, Domingo, Cortijo, Adela (eds.). Valencia: Universitat de València, Facultat de Filologia/ A.P.F.F.U.E., 2001, pp. 157-174.

Ledesma Pedraz, Manuela, "Les échos de la fête dans Madame Bovary de Gustave Flaubert", Écrire, traduire et représenter la fête. VIII Coloquio de la Asociación de Profesores de Filología Francesa de la Universidad Española, Real, Elena, Jiménez, Dolores, Pujante, Domingo, Cortijo, Adela (eds.). Valencia: Universitat de València, Facultat de Filologia/ A.P.F.F.U.E., 2001, pp. 221-229.

MEDINA ARJONA, Encarnación, «La laicización de la fiesta en la literatura naturalista», Écrire, traduire et représenter la fête. VIII Coloquio de la Asociación de Profesores de Filología Francesa de la Universidad Española, Real, Elena, Jiménez, Dolores, Pujante, Domingo, Cortijo, Adela (eds.). Valencia: Universitat de València, Facultat de Filologia/ A.P.F.F.U.E., 2001, pp. 241-252.

Parra Gallardo, Concha, Cuéllar Serrano, Carmen, «El estallido del relato en el capítulo de los comicios agrícolas de Madame Bovary", Écrire, traduire et représenter la fête. VIII Coloquio de la Asociación de Profesores de Filología Francesa de la Universidad Española, Real, Elena, Jiménez, Dolores, Pujante, Domingo, Cortijo, Adela (eds.). Valencia: Universitat de València, Facultat de Filologia/ A.P.F.F.U.E., 2001, pp. 231-240.

REAL, Elena, «Afroditas decadentes», El Fil d'Ariadna. Bari: Ed. Levante Editori, 2001, pp. 379-388.

REAL, Elena, «La fiesta de las lágrimas: el melodrama», Écrire, traduire et représenter la fête. VIII Coloquio de la Asociación de Profesores de Filología Francesa de la Universidad Española, Real, Elena, Jiménez, Dolores, Pujante, Domingo, Cortijo, Adela (eds.). Valencia: Universitat de València, Facultat de Filologia/ A.P.F.F.U.E., 2001, pp. 131-144.

RIPOLL, Ricard, «Le chemin abrupt et sauvage de l'écriture de Lautréamont», Repères \& Applications en didactique des langues, Maynou, Neu, Tost, Manuel A. (eds.). Barcelona: Institut de Ciències de l'Educació, 2001, pp. 180-187.

TAMARIT VALLÉs, Inmaculada, «Les sens de la fête: allez au bal», Écrire, traduire et représenter la fête. VIII Coloquio de la Asociación de Profesores de Filología Francesa de la Universidad Española, Real, Elena, Jiménez, Dolores, Pujante, Domingo, Cortijo, Adela (eds.). Valencia: Universitat de València, Facultat de Filologia/ A.P.F.F.U.E., 2001, pp. 187-195. [Mme de Duras, Édouard].

\section{SigLO XX}

ALbERTINI, Jean, «Revolution/ Revolutions?», Surrealimo y literatura en Europa, Santa, Àngels, Giné, Marta (eds.). Lleida: Edicions de la Universitat de Lleida, 2001, pp. 15-23. 
Álvarez Rubio, M. ${ }^{a}$ del Rosario, «En torno a una interpretación de lo sagrado en el teatro de Henry de Montherlant: La Reine morte (1942)», El teatro y lo sagrado: de M. de Ghelderode a F. Arrabal, Torres Monreal, Francisco (ed.). Murcia: Universidad de Murcia, Servicio de Publicaciones, 2001, pp. 571-580.

ARAGón COBO, Marina, «Françoise Sagan, une sempiternelle fêtarde?», Écrire, traduire et représenter la fête. VIII Coloquio de la Asociación de Profesores de Filología Francesa de la Universidad Española, Real, Elena, Jiménez, Dolores, Pujante, Domingo, Cortijo, Adela (eds.). Valencia: Universitat de València, Facultat de Filologia/ A.P.F.F.U.E., 2001, pp. 483-493.

Badiola Doronsoro, María, «La escritura y el juego en Valery Larbaud», Écrire, traduire et représenter la fête. VIII Coloquio de la Asociación de Profesores de Filología Francesa de la Universidad Española, Real, Elena, Jiménez, Dolores, Pujante, Domingo, Cortijo, Adela (eds.). Valencia: Universitat de València, Facultat de Filologia/ A.P.F.F.U.E., 2001, pp. 431-439.

Benoit Morinière, Claude, «Las Bacantes: Renée Vivien. Poesía y lesbianismo en los albores del siglo XX", Miradas sobre la sexualidad en el arte y la literatura del siglo XX en Francia y en España, Aliaga, Juan Vicente, Haderbache, Ahmed, Monleón, Ana, Pujante, Domingo (eds.). Valencia: Servei de Publicacions de la Universitat de València, 2001, pp. 439-449.

Bermúdez Medina, Dolores, «Hebdomeros de Giorgio de Chirico: le récit mélancolique», Thélème. Revista Complutense de Filología Francesa 16 (2001): 25-33.

Bermúdez Medina, Dolores, LéCrIvain, Claudine, «La métamorphose incessante des brumes: la couleur sentimentale du paysage mirbellien», Cahiers Octave Mirbeau 8 (2001): 34-46.

BERMúdeZ, Lola, «Au verso des mots, les images. Michel Leiris», Surrealimo y literatura en Europa, Santa, Àngels, Giné, Marta (eds.). Lleida: Edicions de la Universitat de Lleida, 2001, pp. 167-177.

Blanariu, Adina, «Le sacré et le profane dans le théàtre de Mircea Eliade», El teatro y lo sagrado: de $M$. de Ghelderode a F. Arrabal, Torres Monreal, Francisco (ed.). Murcia: Universidad de Murcia, Servicio de Publicaciones, 2001, pp. 403409.

CaAmano Piñeiro, M. ${ }^{a}$ Ángeles, «Les fêtes amérindiennes dans l'écriture de Le Clézio», Écrire, traduire et représenter la fête. VIII Coloquio de la Asociación de Profesores de Filologia Francesa de la Universidad Española, Real, Elena, Jiménez, Dolores, Pujante, Domingo, Cortijo, Adela (eds.). Valencia: Universitat de València, Facultat de Filologia/ A.P.F.F.U.E., 2001, pp. 519-526.

Camero Pérez, Carmen, «L'enseveli de J. Schlumberger ou l'écriture d'une voix agonizante», La voix narrative, I, Cahiers de Narratologie (Université de NiceSophia Antipolis) 10: 253-261

Camero Pérez, Carmen, "La subjectividad espacializada. Topos de intimidad en la narrativa de Jacques Henric», Creación espacial y narración literaria, Pérez, 
Concepción, Caballos, M.a de Gracia, Raventós, Anna (eds.). Sevilla: Universidad de Sevilla, Grupo de Investigación Temático Estructural, 2001, pp. 137-140. CASADO, Loreto, "La négation de la fête dans la poésie de Michel Houellebecq", Écrire, traduire et représenter la fête. VIII Coloquio de la Asociación de Profesores de Filología Francesa de la Universidad Española, Real, Elena, Jiménez, Dolores, Pujante, Domingo, Cortijo, Adela (eds.). Valencia: Universitat de València, Facultat de Filologia/ A.P.F.F.U.E., 2001, pp. 575-585.

CAstaño RuIZ, Juana, «El mosaico multiétnico en la obra de Daniel Pennac», Tonos

Digital. Revista electrónica de la Universidad de Murcia, 2001.

Cortijo Talavera, Adela, «Humor negro o sonrisa patafísica. Boris Vian», Humor

i Literatura. Quaderns de Filologia, Estudis Literaris, VI. Valencia: Universitat de València, 2001, pp. 77-89.

Cortuo Talavera, Adela, «La pornografía de Boris Vian en las novelas firmadas Vernon Sullivan», Miradas sobre la sexualidad en el arte y la literatura del siglo XX en Francia y España, Aliaga, Juan Vicente, Haderbache, Ahmed, Monleón, Ana, Pujante, Domingo (eds.). Valencia: Servei de Publicacions de la Universitat de València, 2001, pp. 561-573.

Cortuo TAlavera, Adela, "Swing chez le Major, surprise-party chez Boris Vian», Écrire traduire et représenter la fête. VIII Coloquio de la Asociación de Profesores de Filología Francesa de la Universidad Española, Real, Elena, Jiménez, Dolores, Pujante, Domingo, Cortijo, Adela (eds.). Valencia: Universitat de València, Facultat de Filologia/ A.P.F.F.U.E., 2001, pp. 441-451.

Domínguez LeIva, Antonio, «Decadencia, irracionalismo y violencia: Artaud y el Colegio de Sociología a la sombra de Mein Kampf», Surrealimo y literatura en Europa, Santa, Àngels, Giné, Marta (eds.). Lleida: Edicions de la Universitat de Lleida, 2001, pp. 47-74.

FACio, Ángel, «Ella». El teatro y lo sagrado: de $M$. de Ghelderode a F. Arrabal, Torres Monreal, Francisco (ed.). Murcia: Universidad de Murcia, Servicio de Publicaciones, 2001, pp. 453-457.

Figuerola Cabrol, M. Carme, «Amor y sexo en La Nuit Kurde de Jean-Richard Bloch», Miradas sobre la sexualidad en el arte y la literatura del siglo XX en Francia y España, Aliaga, Juan Vicente, Haderbache, Ahmed, Monleón, Ana, Pujante, Domingo (eds.). Valencia: Servei de Publicacions de la Universitat de València, 2001, pp. 589-604.

Figuerola Cabrol, M. ${ }^{a}$ Carme, «El término oriente en los ensayos de Jean-Richard Bloch», La lingüistica francesa en España camino del siglo XXI, Casal, M. Luz, Conde, Germán, Lago, Jesús, Pino, Laura, Rodríguez, Nuria (eds.). Madrid: Arrecife, 2001, pp. 469-479.

Figuerola Cabrol, M. Carme, «Escribir la fiesta: Le Pont des soupirs y Les amants de Venise de Michel Zévacon, Écrire, traduire et représenter la fête. VIII Coloquio de la Asociación de Profesores de Filología Francesa de la Universidad 
Española, Real, Elena, Jiménez, Dolores, Pujante, Domingo, Cortijo, Adela (eds.). Valencia: Universitat de València, Facultat de Filologia/ A.P.F.F.U.E., 2001, pp. 409-419.

Figuerola CABrol, M. ${ }^{a}$ Carme, «La question coloniale et son influence dans Confidence africaine", Roger Martin du Gard et les crises de l' histoire (colonialisme, seconde guerre mondiale), Daspre, André, Tassel, Alain (eds.). Nice: Presses Universitaires de Nice, 2001, pp. 49-65.

Figuerola CABrol, M. ${ }^{a}$ Carme, «La voix narrative dans le populaire: Le pont des soupirs et Les Amants de Venise de Michel Zévaco», Cahiers de narratologie. La Voix narrative 10/1 (Nice, Publications de la Faculté des Lettres, Arts et Sciences Humaines de Nice) 2001: 417-432.

FoucarT, Claude, «Des poils: surréalisme et esthétique de la laideur (René Crevel)", Surrealimo y literatura en Europa, Santa, Àngels, Giné, Marta (eds.). Lleida: Edicions de la Universitat de Lleida, 2001, pp. 101-112.

García Larrañaga, M. ${ }^{a}$ Asunción, Ibeas Vuelta, Nieves, «Evasión y espíritu carnavalesco en ciencia ficción: la fiesta en la obra de Joëlle Wintrebert», Écrire, traduire et représenter la fête. VIII Coloquio de la Asociación de Profesores de Filología Francesa de la Universidad Española, Real, Elena, Jiménez, Dolores, Pujante, Domingo, Cortijo, Adela (eds.). Valencia: Universitat de València, Facultat de Filologia/ A.P.F.F.U.E., 2001, pp. 537-550.

GarCfA, Mar, «Dispositifs fictionnels dans l'ceuvre fragmentaire de Julien Gracq: récit réaliste et effet de fiction», Fabula (Revista digital). Colloque en ligne 2001 L'effet de fiction. (http://www fabula.org/effet/).

GINE JANER, Marta, «Manifeste du surréalisme: Breton, lector de Villiers», Surrealimo y literatura en Europa, Santa, Àngels, Giné, Marta (eds.). Lleida: Edicions de la Universitat de Lleida, 2001, pp. 89-100.

Gueraud, Jean-François, «Le jeu verbal chez René Crevel», Surrealimo y literatura en Europa, Santa, Àngels, Giné, Marta (eds.). Lleida: Edicions de la Universitat de Lleida, 2001, pp. 115-122.

HAderbache, Ahmed, «Sexe, drogue, séropositivité: un leitmotiv de la fête chez Guillaume Dustan», Écrire, traduire et représenter la fête. VIII Coloquio de la Asociación de Profesores de Filología Francesa de la Universidad Española. Real, Elena, Jiménez, Dolores, Pujante, Domingo, Cortijo, Adela (eds.). Valencia: Universitat de València, Facultat de Filologia/ A.P.F.F.U.E., 2001, pp. 565573.

HARDERBACHE, Ahmed, «Voir et comprendre le Sacré dans Les Bonnes de Genet et La Cérémonie de Claude Chabrol», El teatro y lo sagrado: de $M$. de Ghelderode a F. Arrabal, Torres Monreal, Francisco (ed.). Murcia: Universidad de Murcia, Servicio de Publicaciones, 2001, pp. 411-415.

Hernández Rodriguez, Francisco Javier, «El eje surrealista París-Tenerife», Surrealismo y Literatura en España. Lleida: Universitat de Lleida, 2001, pp. 141-155. 
Illanes ORTEGA, Inmaculada, «Del sentimiento trágico de la fiesta: la España de Paul Morand», Écrire, traduire et représenter la fête. VIII Coloquio de la Asociación de Profesores de Filología Francesa de la Universidad Española, Real, Elena, Jiménez, Dolores, Pujante, Domingo, Cortijo, Adela (eds.). Valencia: Universitat de València, Facultat de Filologia/ A.P.F.F.U.E., 2001, pp. 465-473.

ILlANES ORTEGA, Inmaculada, «Paul Morand nouvelliste: du portrait à l'histoire», $L a$ nouvelle de langue française aux frontières des autres, du Moyen Âge à nos jours. Actes du Colloque de Louvain-la-Neuve, mai 1997, Engel, Vincent, Guissard, Michel (eds.). Louvain-la-Neuve: Academia Bruylant, II, 2001, pp. 201-212.

ILLANES ORTEGA, Inmaculada, «Viajes entre el matrimonio y el deseo: La modification de Michel Butor y La motocyclette de André Pieyre de Mandiargues», Creación espacial y narración literaria, Pérez, Concepción, Caballos, M. ${ }^{\text {a de Gra- }}$ cia, Raventós, Anna (eds.). Sevilla: Universidad de Sevilla, Grupo de Investigación Temático Estructural, 2001, pp. 238-244.

JiMÉNEZ, Dolores, «Sexo, mentiras y anuncios de contacto. Una aproximación heterosexual», Miradas sobre la sexualidad en el arte y la literatura del siglo XX en Francia y España, Aliaga, Juan Vicente, Haderbache, Ahmed, Monleón, Ana, Pujante, Domingo (eds.). Valencia: Servei de Publicacions de la Universitat de València, 2001, pp. 79-90

JOVER SilveSTRE, Yolanda, «Si je meurs je veux qu'on m'enterre dans une cave ou y'a du bon vin», Écrire, traduire et représenter la fête. VIII Coloquio de la Asociación de Profesores de Filología Francesa de la Universidad Española, Real, Elena, Jiménez, Dolores, Pujante, Domingo, Cortijo, Adela (eds.). Valencia: Universitat de València, Facultat de Filologia/ A.P.F.F.U.E., 2001, pp. 527-536.

LEAL, Juli, «Referentes icónicos de lo sagrado en el montaje de Las criadas de Genet realizado por Víctor García-Nuria Espert», El teatro y lo sagrado: de M. de Ghelderode a F. Arrabal, Torres Monreal, Francisco (ed.). Murcia: Universidad de Murcia, Servicio de Publicaciones, 2001, pp. 417-429.

LiCCIOLI, Edi, «La recuperación del mimo fúnebre en Genet», El teatro y lo sagrado: de $M$. de Ghelderode a F. Arrabal, Torres Monreal, Francisco (ed.). Murcia: Universidad de Murcia, Servicio de Publicaciones, 2001, pp. 431-439.

Mallart Brussosa, Myriam, «L'étrange fête du Grand Meaulnes», Écrire, traduire et représenter la fête. VIII Coloquio de la Asociación de Profesores de Filología Francesa de la Universidad Española, Real, Elena, Jiménez, Dolores, Pujante, Domingo, Cortijo, Adela (eds.). Valencia: Universitat de València, Facultat de Filologia/ A.P.F.F.U.E., 2001, pp. 421-430.

Michaud, Stéphane, «Je suis Nietzsche..., Bretón intérprete de Nietzsche», Surrealimo y literatura en Europa, Santa, Àngels, Giné, Marta (eds.). Lleida: Edicions de la Universitat de Lleida, 2001, pp. 75-87.

MONLEÓN DOMfNGUEZ, Ana, «Las escrituras lesbianas», Miradas sobre la sexualidad en el arte y la literatura del siglo XX en Francia y España, Aliaga, Juan Vicen- 
te, Haderbache, Ahmed, Monleón, Ana (eds.). Valencia: Servei de Publicacions de la Universitat de València, 2001, pp. 467-482.

MOUNTASAR, Rachid, «Image verbale et incarnation dans Les Bonnes de Jean Genet», El teatro y lo sagrado: de $M$. de Ghelderode a F. Arrabal, Torres Monreal, Francisco (ed.). Murcia: Universidad de Murcia, Servicio de Publicaciones, 2001, pp. 441-452.

PARDo Jiménez, Pedro, «Marcel Aymé, ou l'art d'abréger», La nouvelle de langue française aux frontières des autres genres, du Moyen Âge à nos jours. Actes du Colloque de Louvain-La-Neuve, mai 1997, Engel, V., Guissard, M. (eds.). Louvain-La-Meuve: Academia Bruylant, II, 2001, pp. 245-252.

Peñalva García, M. E., «Ensoñación de los amores sáficos: En déharquant à Mytilène de Renée Vivien", Poesía del mundo francófono contemporáneo. Antología crítica, Grupo de Investigación Traducción y Análisis Literarios. Sevilla: Ed. Kronos/ Departamento de Filología Francesa, 2001, pp. 49-63.

Peñalva García, M. E., «La cosmicidad: espacio mítico de ensoñación aérea en Les Météores de Michel Tournier», Creación espacial y narración literaria, Pérez, Concepción, Caballos, M. ${ }^{a}$ de Gracia, Raventós, Anna (eds.). Sevilla: Universidad de Sevilla, Grupo de Investigación Temático Estructural, 2001, pp. 329-337

Pérez LaCarta, Ana María, "Les jardins et la forêt du Dieu nu», Cahiers Rohert Margerit 5 (2001): 10-19. [Margerit].

Piquer Desvaux, Alicia, "L'évolution du fantastique dans les nouvelles de Louis Aragon», La nouvelle de langue française aux frontières des autres genres, du Moyen Âge à nos jours. Actes du Colloque de Louvain-la-Neuve, mai 1997, Engel, Vincent, Guissard, Michel (eds.). Louvain-la-Neuve: Academia Bruylant. 2001, pp. 258-267.

PIQUER, Alicia, «L'écriture poétique au $\mathrm{XX}^{e}$ siècle», Introducció a la literatura francesa, Cots, Montserrat (ed.). Barcelona: Universitat Oberta de Catalunya, 2001, pp. 5-27. Piquer, Alicia, «L'évolution du fantastique dans les nouvelles de Louis Aragon", La nouvelle de langue française aux frontières des autres genres, du Moyen Ầge à nos jours. Actes du Colloque de Louvain-la-Neuve, mai 1997. Engel, Vincent, Guissard, Michel (eds.). Louvain-la-Neuve: Academia-Bruylant, II, 2001, pp. 213-223.

PujANTE GonzÁlez, Domingo, «Cy Jung: la revindicación de lo sexual femenino a través de la escritura", Miradas sobre la sexualidad en el arte y la literatura del siglo XX en Francia y España, Aliaga, Juan Vicente, Haderbache, Ahmed, Monleón, Ana (eds.). Valencia: Servei de Publicacions de la Universitat de València, 2001, pp. 451 ss.

PujAnte GonZález, Domingo, «El humor en la obra de Roland Topor: la risa invertida como revulsivo social», Humor i Literatura. Quaderns de Filologia, Estudis Literaris, VI. Valencia: Universitat de València, 2001, pp. 173-184.

PujANTE GonZÁleZ, Domingo, «Roland Topor y Alejandro Jodorowsky: dos farsantes pánicos, dos burlones del orden", Estudios sobre humor literario, Figueroa, 
J., Urdiales, M., Larkin, C., Vázquez, C. (eds.). Vigo: Universitade de Vigo, 2001, pp. 351-359.

Pujante GonZález, Domingo, «Sacrificio y transgresión: la supervivencia de lo sagrado en las artes escénicas de los sesenta», El teatro y lo sagrado: de $M$. de Ghelderode a F. Arrabal, Torres Monreal, Francisco (ed.). Murcia: Universidad de Murcia, Servicio de Publicaciones, 2001, pp. 161-177.

REAL, Elena, «La creación del espacio surrealista», Surrealimo y literatura en Europa, Santa, Àngels, Giné, Marta (eds.). Lleida: Edicions de la Universitat de Lleida, 2001, pp. 133-145.

REAL, Elena, «Michel Leiris: alegorías del sentimiento trágico de la sexualidad», Miradas sobre la sexualidad en el arte y la literatura del siglo XX en Francia y España, Aliaga, Juan Vicente, Haderbache, Ahmed, Monleón, Ana (eds.). Valencia: Servei de Publicacions de la Universitat de València, 2001, pp. 549-560.

Rufat PERelló, Hélène, «Des paradis au rite: les fètes camusiennes», Écrire, traduire et représenter la fête. VIII Coloquio de la Asociación de Profesores de Filología Francesa de la Universidad Española, Real, Elena, Jiménez, Dolores, Pujante, Domingo, Cortijo, Adela (eds.). Valencia: Universitat de València, Facultat de Filologia/ A.P.F.F.U.E., 2001, pp. 453-463.

SALCEDA, Hermes, «La reconstrucción de la memoria en La Disparition de Georges Perec", Écrire, traduire et représenter la fête. VIII Coloquio de la Asociación de Profesores de Filología Francesa de la Universidad Española, Real, Elena, Jiménez, Dolores, Pujante, Domingo, Cortijo, Adela (eds.). Valencia: Universitat de València, Facultat de Filologia/ A.P.F.F.U.E., 2001, pp. 495-504.

SANTA, Àngels, USALL, Ramon, «Existencialismo y comunismo frente a surrealismo: de Nizan a Sartre», Surrealimo y literatura en Europa, Santa, Àngels, Giné, Marta (eds.). Lleida: Edicions de la Universitat de Lleida, 2001, pp. 35-45.

SERRANO MAÑES, Monserrat, «La fête des mots: de l'amertume au rire par la transgression verbale», Écrire, traduire et représenter la fête. VIII Coloquio de la Asociación de Profesores de Filología Francesa de la Universidad Española, Real, Elena, Jiménez, Dolores, Pujante, Domingo, Cortijo, Adela (eds.). Valencia: Universitat de València, Facultat de Filologia/ A.P.F.F.U.E., 2001, pp. 383-396.

SOLÀ, Pere, «El llenguatge de l'Aragon dadaista i surrealista», Surrealimo y literatura en Europa, Santa, Àngels, Giné, Marta (eds.). Lleida: Edicions de la Universitat de Lleida, 2001, pp. 123-131.

SolÀ, Pere, «Las fiestas de Prévert», Écrire, traduire et représenter la fête. VIII Coloquio de la Asociación de Profesores de Filología Francesa de la Universidad Española, Real, Elena, Jiménez, Dolores, Pujante, Domingo, Cortijo, Adela (eds.). Valencia: Universitat de València, Facultat de Filologia/ A.P.F.F.U.E., 2001, pp. 475-482.

SolÉ CASTElls, Cristina, «Henri Barbusse y el surrealismo», Surrealimo y literatura en Europa, Santa, Àngels, Giné, Marta (eds.). Lleida: Edicions de la Universitat de Lleida, 2001, pp. 25-32. 
SuÁrez SÁNCHEZ, Elena, «Las riberas de una ensoñación», Creación espacial y narración literaria, Pérez, Concepción, Caballos, M. ${ }^{a}$ de Gracia, Raventós, Anna (eds.). Sevilla: Universidad de Sevilla, Grupo de Investigación Temático Estructural, 2001, pp. 364-369.

SuÁrez SÁnChEz, Elena, «Le paysage de Robert Desnos o de la transgresión a la norma», Poesía del mundo francófono contemporáneo. Antología crítica, Grupo de Investigación Traducción y Análisis Literarios. Sevilla: Ed. Kronos/ Departamento de Filología Francesa, 2001, pp. 131-145.

Thomas, Nadine, «Le sens de la fête chez Le Clézio», Écrire, traduire et représenter la fête. VIII Coloquio de la Asociación de Profesores de Filología Francesa de la Universidad Española, Real, Elena, Jiménez, Dolores, Pujante, Domingo, Cortijo, Adela (eds.). Valencia: Universitat de València, Facultat de Filologia/ A.P.F.F.U.E., 2001 , pp. 505-518.

TORRES MONREAl, Francisco, «El teatro y lo sagrado: de 1930 a $2000 »$, El teatro y lo sagrado: de $M$. de Ghelderode a $F$. Arrabal, Torres Monreal, Francisco (ed.). Murcia: Universidad de Murcia, Servicio de Publicaciones, 2001, pp. 15-62.

Vilvandre DE SousA, Cécile, «El Rey se muere, de Eugène Ionesco: un rito iniciático sacralizador de la muerte", El teatro y lo sagrado: de $M$. de Ghelderode a $F$. Arrabal, Torres Monreal, Francisco (ed.). Murcia: Universidad de Murcia, Servicio de Publicaciones, 2001, pp. 581-589.

\section{VARIOS SIGLOS}

Cots, Montserrat (ed.), Introducció a la literatura francesa. Barcelona: Universitat Oberta de Catalunya, 2001.

GonzÁlez SAlvador, Ana, "Revers de l'hospitalité: vampirisme», Lieux d'hospitalité. Hospices, hôpital, hostellerie, Montandon, Alain (ed.). Clermont-Ferrand: Presses Universitaires, Université Blaise-Pascal, 2001, pp. 487-496.

TOMAS, Ilda, «Provocation, badinage et liberté à travers les Fêtes galantes et les $F \hat{e}$ tes foraines", Écrire, traduire et représenter la fête. VIII Coloquio de la Asociación de Profesores de Filología Francesa de la Universidad Española, Real, Elena, Jiménez, Dolores, Pujante, Domingo, Cortijo, Adela (eds.). Valencia: Universitat de València, Facultat de Filologia/ A.P.F.F.U.E., 2001, pp. 253-262.

9. LITERATURA DE EXPRESIÓN FRANCESA. LITERATURA EN LENGUA FRANCESA FUERA DE FRANCIA

Alfaro, Margarita, «Evolución de la poesía en Quebec», Literatura francocanadiense: la literatura quebequesa, Fernández Sánchez, Carmen (ed.). Oviedo: Universidad de Oviedo, Servicio de Publicaciones, 2001, pp. 215-286. 
ANOLL, Lidia, «El relato breve en Quebec», Literatura francocanadiense: la literatura quebequesa, Fernández Sánchez, Carmen (ed.). Oviedo: Universidad de Oviedo, Servicio de Publicaciones, 2001, pp. 104-139.

ANOLL, Lídia, «Introducció i traducció» de Anne Hébert, Les chambres de bois. Lleida: Pagés editors, 2001, 157 pp.

ANOLL, Lídia, «Tournée d'automne amb sol primaveral i lluors de capvespre», $\mathrm{Ho}$ menatge a Antoni Vicens i Castanyer. Palma de Mallorca: Estudi General Lul-lià, 2001, pp. 33-38. [Jacques Poulin, La tournée d'automne (Québec)].

Caballos Bejano, M. ${ }^{\text {a }}$ de Gracia, «La ensoñación del desarraigo en L'Enfant Noir», Más allá de un milenio: globalización, identidades y universos simbólicos. Sevilla: Alfar/ Universidad, 2001, pp. 127-134.

Caballos Bejano, M..$^{a}$ de Gracia, «La mujer negra antillana: percepción social y ensoñación literaria», Representar-representarse. Firmado: Mujer. Congreso Internacional en homenaje a Zenobia Camprubí. Huelva: Fundación Juan Ramón Jiménez, 2001, pp. 141-152.

Cuasante Fernández, Elena, "Juletane o la otra, la extranjera», Francofonía 10 (2001): 21-35.

De la TORRE GIMÉnEZ, Estrella, «Le regard de Marcel Lecomte. À la recherde de l'essence des choses», Mélusine 21 (2001): 125-132.

DE LA TORRE GiménEZ, Estrella, «Un hétérodoxe du surréalisme du Hainaut: Constant Malva», Surrealimo y literatura en Europa, Santa, Àngels, Giné, Marta (eds.). Lleida: Edicions de la Universitat de Lleida, 2001, pp. 179-189.

DEChamPS, Christina, «Ghelderode: la mort et la religion», El teatro y lo sagrado: de $M$. de Ghelderode a F. Arrabal, Torres Monreal, Francisco (ed.). Murcia: Universidad de Murcia, Servicio de Publicaciones, 2001, pp. 471-479.

DíaZ NARbona, Inmaculada, LÉCRIVAIN, Claudine, «Imana, el dios de todos. Ruanda desde la perspectiva de Véronique Tadjo», Extramuros (Granada) 21 (2001): 43-46

DIAZ NARBONA, Inmaculada, «Entre tradition et modernité : Karim d'Ousmane Socé Diop», International Journal of Francophone Studies (Leeds) 4/ 1 (2001): 2432.

Díaz NARBOna, Inmaculada, «Une lecture à rebrousse temps de l'œuvre de Ken Bugul. Critique féministe, critique africaniste», Études françaises (Montreal) 37/ 2 (2001): 115-131

Díaz Narbona, Inmaculada, CuASANTE FernándEZ, Elena, «Firmado mujer y africana. La literatura africana en femenino: un proceso de recuperación de la palabra», Representar-representarse. Firmado: Mujer. Congreso Internacional en homenaje a Zenobia Camprubí. Huelva: Fundación Juan Ramón Jiménez, 2001, pp. 401-412.

FERnÁNDEZ SÁnchEZ, Carmen, «Elementos trágicos y violencia sagrada en el teatro de Michel Tremblay», El teatro y lo sagrado: de $M$. de Ghelderode a F. Arra- 
bal, Torres Monreal, Francisco (ed.). Murcia: Universidad de Murcia, Servicio de Publicaciones, 2001, pp. 521-535.

FERNÁNDEZ SÁNCHEZ, Carmen, "Novela y sociedad en Quebec», Literatura francocanadiense: la literatura quebequesa, Fernández Sánchez, Carmen (ed.). Oviedo: Universidad de Oviedo, Servicio de Publicaciones, 2001, pp. 9-101.

Figueiras LoREnzo, Marta, «El mito de Don Juan en la literatura belga: Charles Bertin y Suzanne Lilar frente a Tirso de Molina», Anuario de Estudios Filológicos (Universidad de Extremadura) 24 (2001): 107-122.

FRANÇOIS FILLEUL, François, «Paul Emond ou le plaisir du topos: bagatelles, galopades, galipettes», Écrire, traduire et représenter la fête. VIII Coloquio de la Asociación de Profesores de Filología Francesa de la Universidad Española. Real, Elena, Jiménez, Dolores, Pujante, Domingo, Cortijo, Adela (eds.). Valencia: Universitat de València, Facultat de Filologia/ A.P.F.F.U.E., 2001, pp. 551-563. GaRCíA, Mar, «Critique littéraire et africanité: dialectique et perspectives», Repères \& Applications en didactique des langues, Maynou, Neu, Tost, Manuel A. (eds.). Barcelona: Institut de Ciències de l'Educació, 2001, pp. 157-166.

GonzÁlez SAlvador, Ana, "L'insolite de l'écriture: la peine du style (À propos du Jardin malade de Michel de Ghelderode)», Thélème. Revista Complutense de Estudios Franceses 16 (2001): 61-69.

GonZÁleZ SAlVADOR, Ana, «Le polar belge: dernières tendances», Les Lettres helges au présent, Lope, Hans-Joachim, Neuschäfer, Anne, Quaghebeur, Marc (eds), Studien und Dokumente zur Geschichte der Romanischen Literaturen, 44. Frankfurt am Main: Peter Lang, 2001, pp. 299-307.

González Salvador, Ana, «Machine d'enfer: Crommelynck et la scène (du crime)», Le Diable en Belgique. Bolonia: CLUEB, 2001, pp. 149-162.

L'HÉRAULT, Pierre, «El teatro quebequés del siglo XX», Literatura francocanadiense: la literatura quebequesa, Fernández Sánchez, Carmen (ed.). Oviedo: Universidad de Oviedo, Servicio de Publicaciones, 2001, pp. 141-214.

LÉCrIvain, Claudine, Traducción de Henri Lopès, Le pleurer-Rire, Présence Africaine, 1982. Ediciones del Bronce, 2001, 315 páginas.

LEQUIN, Lucie, «El ensayo en Quebec», Literatura francocanadiense: la literatura quebequesa, Fernández Sánchez, Carmen (ed.). Oviedo: Universidad de Oviedo, Servicio de Publicaciones, 2001, pp. 287-338.

LEUWERS, Daniel, «Trois acteurs, un drame, de Michel de Ghelderode, ou la désacralisation de l'auteur», El teatro y lo sagrado: de $M$. de Ghelderode a $F$. Arrabal, Torres Monreal, Francisco (ed.). Murcia: Universidad de Murcia, Servicio de publicaciones, 2001, pp. 481-486.

MalingRet, Laurence, «Formules et magie dans le théâtre de Paul Willems», El teatro y lo sagrado: de $M$. de Ghelderode a F. Arrabal, Torres Monreal, Francisco (ed.). Murcia: Universidad de Murcia, Servicio de Publicaciones, 2001, pp. 495-503. 
Michel, Geneviève, «René Magritte et la métaphore transfigurée», Écrire, traduire et représenter la fête. VIII Coloquio de la Asociación de Profesores de Filología Francesa de la Universidad Española. Real, Elena, Jiménez, Dolores, Pujante, Domingo, Cortijo, Adela (eds.). Valencia: Universitat de València, Facultat de Filologia/ A.P.F.F.U.E., 2001, pp. 303-315.

Perales Gutiérrez, J. J., «La poesía: un espacio de solidaridad en Les Chapelets d'attache de Tahar Bekri», Poesía del mundo francófono contemporáneo. Antología crítica, Grupo de Investigación Traducción y Análisis Literarios. Sevilla: Ed. Kronos/ Departamento de Filología Francesa, 2001, pp. 65-87.

Porras Medrano, Adelaida, Baena Gallé, Violeta, «Espacio y desmitificación en La Répudiation de Rachid Boudjedra", Creación espacial y narración literaria, Pérez, Concepción, Caballos, M. ${ }^{a}$ de Gracia, Raventós, Anna (eds.). Sevilla: Universidad de Sevilla, Grupo de Investigación Temático Estructural, 2001, pp. 83-89.

Porras Medrano, Adelaida, «' Octobre de Gaston Miron o la poética de la rebeldía», Poesía del mundo francófono contemporáneo. Antología crítica, Grupo de Investigación Traducción y Análisis Literarios. Sevilla, Ed. Kronos/ Departamento de Filología Francesa, 2001, pp. 89-104.

Porras MEdrano, Adelaida, «La novela», Introducción a la literatura canadiense francófona. Málaga: Analecta Malacitana, Anejo XXXIX, 2001, pp. 223-268.

Porras Medrano, Adelaida, «Polyphonie narrative et récit d'enfance: Le Fils du pauvre de Mouloud Feraoun», Cahiers de Narratologie (Université de NiceSophia Antipolis) 10/2 (2001): 375-382.

PujANTE GonZALEZ, Domingo, «Juego ceremonial, fiesta cruel: del Living Theatre a Fernando Arrabal», Écrire, traduire et représenter la fête. VIII Coloquio de la Asociación de Profesores de Filología Francesa de la Universidad Española, Real, Elena, Jiménez, Dolores, Pujante, Domingo, Cortijo, Adela (eds.). Valencia: Universitat de València, Facultat de Filologia/ A.P.F.F.U.E., 2001, pp. $397-$ 408.

Ripoll, Ricard, «L'écriture de l'exil, ou l'angoisse du vide: une lecture du roman Ana Non d'Agustín Gómez-Arcos», Mots Pluriels (Revista digital) 17 (abril 2001), número dedicado a Écrire l'exil: rupture et continuité (http://www.arts.uwa.edu.au/MotsPluriels/MP.html)

SPECKENS, Rose-Marie, «Trilingüismo y plegaria en el teatro de Ghelderode», El teatro y lo sagrado: de $M$. de Ghelderode a F. Arrabal, Torres Monreal, Francisco (ed.). Murcia: Universidad de Murcia, Servicio de Publicaciones, 2001, pp. 487-494.

Teissier, Guy, «Jésus à Montreal: Figures christiques dans le théâtre québécois», $E l$ teatro y lo sagrado: de M. de Ghelderode a F. Arrabal, Torres Monreal, Francisco (ed.). Murcia: Universidad de Murcia, Servicio de Publicaciones, 2001, pp. 537-554. 
Valén, Antón, Noguera, Roberto, «Michel de Ghelderode: lo divino y lo humano», El teatro y lo sagrado: de $M$. de Ghelderode a F. Arrabal, Torres Monreal, Francisco (ed.). Murcia: Universidad de Murcia, Servicio de Publicaciones, 2001, pp. 505-512.

10. LITERATURA COMPARADA. RECEPCIÓN LITERARIA

AlCalde OnRubia, M. ${ }^{a}$ Paz, «El gusto por lo español en el umbral del siglo XVII francés: la mística teresiana", Écrire, traduire et représenter la fête. VIII Coloquio de la Asociación de Profesores de Filología Francesa de la Universidad Española, Real, Elena, Jiménez, Dolores, Pujante, Domingo, Cortijo, Adela (eds.). Valencia: Universitat de València, Facultat de Filologia/ A.P.F.F.U.E., 2001, pp. 121-129.

Alonso Seoane, M. ${ }^{a}$ José, «Una novela inédita de Olavide y su original francés», $R e$ cepción de autores franceses de la época clásica en los siglos XVIII y XIX en España y en el extranjero, Boixareu, Mercedes, Desné, Roland (eds.), Madrid, UNED, 2001, pp. 247-252.

BitToun, Nathalie, «Le théâtre de Marivaux en Espagne (XVIII ${ }^{c}$ et XIX' siècles)», Recepción de autores franceses de la época clásica en los siglos XVIII y XIX en España y en el extranjero, Boixareu, Mercedes, Desné, Roland (eds.). Madrid: UNED, 2001, pp. 191-204.

CARNERO, Guillermo, «El modelo francés en el teatro, la novela y las misceláneas españolas de finales del XVIII y comienzos del XIX», Recepción de autores franceses de la época clásica en los siglos XVIII y XIX en España y en el extranje. ro, Boixareu, Mercedes, Desné, Roland (eds.). Madrid: UNED, 2001, pp. 25-36. GINE, Giné, «La palabra de Voltaire en El jardín de las dudas de Fernando Savater», Los clásicos franceses en la España del siglo XX. Estudio de traducción y recepción, Lafarga, Francisco, Domínguez, Antonio (eds.). Barcelona: P.P.U., 2001, pp. 237-248.

HuMPERS, Jean-Paul, «Ghelderode-Arrabal: du roi fou à l'empereur dément», El teatro y lo sagrado: de $M$. de Ghelderode a $F$. Arrabal, Torres Monreal, Francisco (ed.). Murcia: Universidad de Murcia, Servicio de Publicaciones, 2001, pp. 459469.

Lafarga, Francisco, «Racine, P. de Olavide, R. Chacel: dos versiones de Phèdre a dos siglos de distancia», Los clásicos franceses en la España del siglo XX. Estudios de traducción y recepción, Lafarga, Francisco, Domínguez, Antonio (eds.). Barcelona: PPU, 2001, pp. 107-116.

Negueruela Ceballos, Jacinta, Navarro Torres, J. Carles, «Yves Bonnefoy y Miquel Barceló: ut pictura poesis», Écrire, traduire et représenter la fête. VIII Coloquio de la Asociación de Profesores de Filología Francesa de la Universidad 
Española, Real, Elena, Jiménez, Dolores, Pujante, Domingo, Cortijo, Adela (eds.). Valencia: Universitat de València, Facultat de Filologia/ A.P.F.F.U.E., 2001, pp. 317-322.

OzAETA, Rosario, «Las fábulas de La Fontaine en el siglo XIX español», Recepción de autores franceses de la época clásica en los siglos XVIII y XIX en España y en el extranjero, Boixareu, Mercedes, Desné, Roland (eds.). Madrid: UNED, 2001, pp. 91-99.

Palacios Bernal, Concepción, «Acerca de la traducción de las Nouvelles de Florian a finales del siglo XVIII», Recepción de autores franceses de la época clásica en los siglos XVIII y XIX en España y en el extranjero, Boixareu, Mercedes, Desné, Roland (eds.). Madrid: UNED, 2001, pp. 237-246.

RAMOS GAY, Ignacio, «La parodia shakespeariana como sumisión y liberación: el caso de Jarry, Ionesco y Stoppard», Estudios sobre el humor literario, Figueroa, J., Urdiales, M., Larkin, C., Vázquez, C. (eds.). Vigo: Universidade de Vigo, 2001, pp. 343-351.

RAMOS GAY, Ignacio, «Sexualidades fragmentadas: lo apolíneo y lo dionisíaco en L'Immoraliste de André Gide y La Muerte en Venecia de Thomas Mann», Miradas sobre la sexualidad en el arte y la literatura del siglo XX en Francia y en España, Aliaga, Juan Vicente, Haderbache, Ahmed, Monleón, Ana, Pujante, Domingo (eds.). Valencia: Servei de Publicacions de la Universitat de València, 2001, pp. 605-616.

Romero Alfaro, Elena, «Mme Leprince de Beaumont: aproximaciones a una recepción española (siglos XVIII y XIX)», Recepción de autores franceses de la época clásica en los siglos XVIII y XIX en España y en el extranjero, Boixareu, Mercedes, Desné, Roland (eds.), Madrid, UNED, 2001, pp. 321-329.

SÁNCHEZ EsPINOSA, Gabriel, «Mme de Sévigné y la carta epistolar en España durante el siglo XVIII», Recepción de autores franceses de la época clásica en los siglos XVIII y XIX en España y en el extranjero, Boixareu, Mercedes, Desné, Roland (eds.). Madrid: UNED, 2001, pp. 111-123.

SANTOYO, Julio César, «Traducción y reescrituras: el paradigma de las Lettres persanes de Montesquieu», Recepción de autores franceses de la época clásica en los siglos XVIII y XIX en España y en el extranjero, Boixareu, Mercedes, Desné, Roland (eds.). Madrid: UNED, 2001, pp. 37-47.

SANZ, Amelia, «Les genres narratifs du XVII' siècle français en Espagne: un refus de lecture», Recepción de autores franceses de la época clásica en los siglos XVIII y XIX en España y en el extranjero, Boixareu, Mercedes, Desné, Roland (eds.). Madrid: UNED, 2001, pp. 227-236.

SAURA SÁNCHEZ, Alfonso, «La narrativa francesa en Lunes de papel», Estudios Románicos (Universidad de Murcia) 12 (2001): 225-240.

SAURA SÁnchez, Alfonso, «Recepción en España de las tragedias de Prosper Jolyot de Crébillon", Recepción de autores franceses de la época clásica en los siglos 
XVIII y XIX en España y en el extranjero, Boixareu, Mercedes, Desné, Roland (eds.). Madrid: UNED, 2001, pp. 171-190.

Tol.IVAR, Ana Cristina, «La réception de Racine dans l'Espagne du XVIII" siècle», Recepción de autores franceses de la época clásica en los siglos XVIII y XIX en España y en el extranjero. Boixareu, Mercedes, Desné, Roland (eds.). Madrid: UNED, 2001, pp. 127-137.

TORRes MONREAL. Francisco, «Calderón de la Barca en el teatro francés. 19301973», Calderón protagonista del Barroco europeo, Reinchenberger, Kurt y Theo (eds.), Kassel, Reichemberger, 2001, pp. 569-606

Torres Monreal, Francisco, «La Célestine en el teatro francés (s. XX): de P. Achard a Jean Gillibert», Tras los pasos de La Celestina, Botta, P., Catalapiedra, F., Reichenberger, K, Snow, J. T. Snow (eds.), Kassel, Reichenberger, 2001, pp. 315-344.

11. ESTUdios SOBRE LA TRADUCCIÓN LITERARIA

ANOLl, Lídia, «Acerca de la traducción de unas cartas de Madame du Deffand a Voltaire», Los clásicos franceses en la España del siglo XX. Estudios de traducción y recepción, Lafarga, Francisco, Domínguez, Antonio (eds.). Barcelona: PPU, 2001 , pp. 221-227.

Camero Pérez, Carmen, «De l'anthologie au recueil: structure et organisation du livre de nouvelles", La nouvelle de langue française aux frontières des autres, du Moyen Âge à nos jours. Actes du Colloque de Louvain-la-Neuve, mai 1997. Engel, Vincent, Guissard, Michel (eds.). Louvain-la-Neuve: Academia Bruylant, II, 2001, pp. 396-401

GINE, Marta, «El teatre du bon sens en català: Dumas fill», La traducción en la Edad de Plata, Pegenaute, Luis (ed.). Barcelona: PPU, 2001, pp. 75-88.

LAFARGa, Francisco, "Teodoro Llorente, traductor y antólogo de poesía francesa", La traducción en la Edad de Plata, Pegenaute, Luis (ed.). Barcelona: PPU, 2001, pp. 157-169.

Lécrivain, Claudine, Romero Alfaro, Elena, «La synesthésie déconstruite ou l'intermédiaire traductionnel sourd au bruit des couleurs et insensible au goût de la musique», Meta. Revue des Traducteurs 46 (2001): 524-534.

MARTíneZ, Caridad, "Traducir a Louise Labé», Los clásicos franceses en la España del siglo XX. Estudios de traducción y recepción. Lafarga, Francisco, Domínguez, Antonio (eds.). Barcelona: PPU, 2001, pp. 263-272.

Palacios Bernal, Concepción, "Molière traducido por Gómez de la Serna». Los clásicos franceses en la España del siglo XX. Estudios de traduc'ión y recepción, Lafarga, Francisco, Domínguez, Antonio (eds.). Barcelona: PPU, pp. 129 138. 
Piquer Desvaux, Alicia, «Jorge Guillén, traductor de Supervielle», La traducción en la Edad de Plata. Pegenaute, Luis (ed.). Barcelona: PPU, 2001, pp. 259-270.

Piquer Desvaux. Alicia, «Traducción y recepción de Ronsard y otros poetas de su tiempo", Los clásicos franceses en la España del siglo XX. Estudios de traducción y recepción, Lafarga, Francisco, Domínguez. Antonio (eds.). Barcelona: PPU, 2001, pp. 57-65.

SANTA, Àngels, CourT, Antoine, «Familia y clases populares en las traducciones de Geneviève de Lamartine durante el siglo XIX». Historia social y literatura. Familia y clases populares en España (siglos XVIII-XIX), Fernández, Roberto, Soubeyroux. Jacques (eds.). Editorial Milenio/ Université Jean Monnet, SaintÉtienne, 2001. pp. 255-266.

\section{Historia de las ideas literarias. Crítica literaria. Géneros}

RIPOL.L. Ricard, «L'aventura du fictif», Fabula (Revista digital). Colloque en ligne 2001 L'effet de fiction. ( http://n'n'w fabula.org/effet/).

\section{Literatura OCCITANA}

Blanco Garcla, Pilar, «La literatura en busca de la toponimia», Érire, traduire et représenter la fête. VIII Coloquio de la Asociación de Profesores de Filología Francesa de la Universidad Española, Real, Elena, Jiménez. Dolores, Pujante. Domingo, Cortijo, Adela (eds.). Valencia: Universitat de València, Facultat de Filologia/ A.P.F.F.U.E., 2001, pp. 263-272.

Día7. Cerectida. Araceli, «Soporte cultural de la lírica trovadoresca», Écrire, traduirét représenter la fête. VIII Coloquio de la Asociación de Profesores de Filología Francesa de la Universidad Española. Real. Elena, Jiménez, Dolores, Pujante, Domingo, Cortijo, Adela (eds.). Valencia: Universitat de València, Facultat de Filologia/ A.P.F.F.U.E., 2001, pp. $71-77$.

Mot.To. Elena, «Entre el rito y el juego: la fiesta cortés», Érire, traduire et représenter la fête. VIII Coloquio de la Asociación de Profesores de Filología Franresa de la Universidad Española, Real, Elena, Jiménez, Dolores. Pujante. Domingo, Cortijo, Adela (eds.). Valencia: Universitat de València, Facultat de Filologia/ A.P.F.F.U.E., 2001, pp. 79-88.

Mol.to, Elena, «Riure's dels altres i riure's amb els altres (sobre el sentit de l'humor occità)". Humor i Literatura. Quaderns de Filologia, Estudis Literaris. VI. Gregori, Carmen. Jiménez, Dolores. Martínez Luciano, Juan Vicente (eds.). Valencia: Facultat de Filologia, Universitat de València, 2001, pp. $119-128$. 


\section{ENSEÑANZA DE LA LiTERATURA}

Martin, Christophe, «Éloge de la diversalité. Sur l'enseignement des littératures de langue française», Repères \& Applications en didactique des langues, Maynou, Neu, Tost, Manuel A. (eds.). Barcelona: Institut de Ciències de l'Educació, 2001, pp. 167-179.

15. Bibliografía

YLLERA, A.: «Publicaciones sobre literatura francesa en España (2000)», Epos 17 (2001), pp. 457-474. 